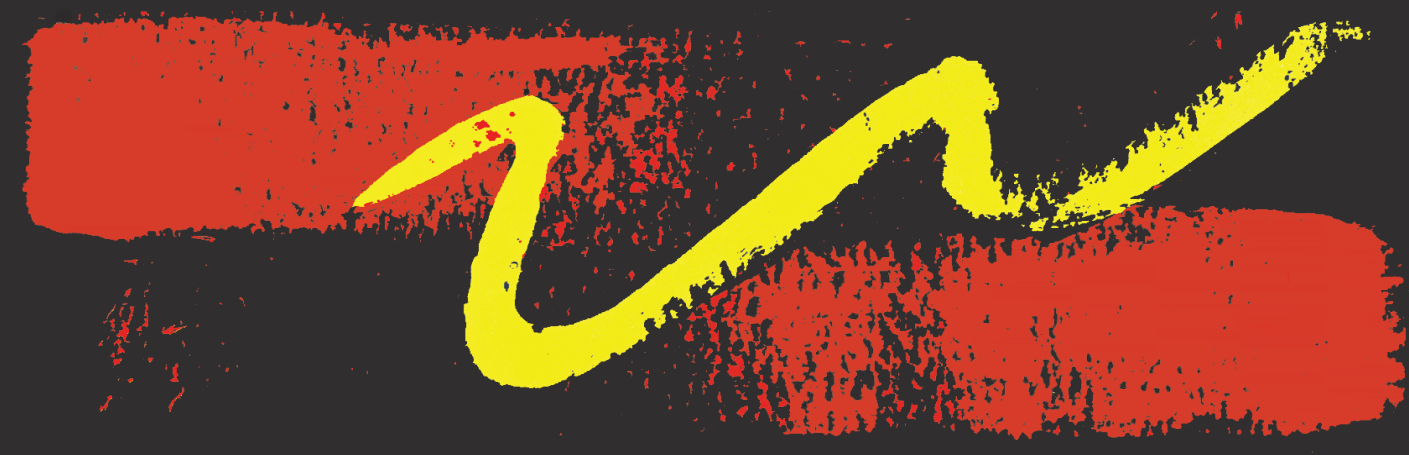

Vol. XXV (2), 2007

ISSN 0254-9247
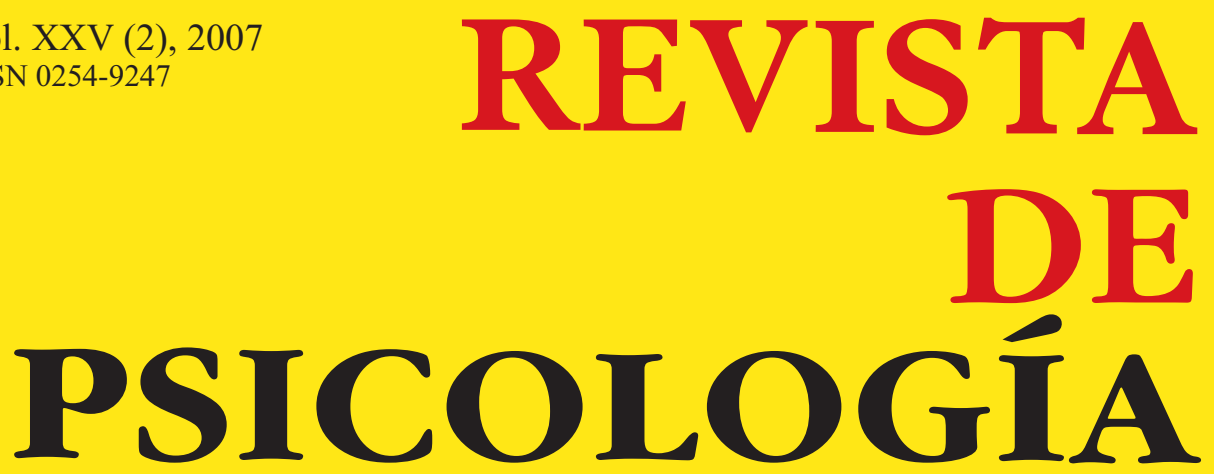

Ana María Anguas-Wong

María Ángeles Bilbao

Gloria Burga

Alicia Calderón-Prada

Agustín Espinosa

Ana Rosa Feijoo

Jessica Güímac

Rom Harré

Naomi Lee

David Matsumoto

Fathali M. Moghaddam

Darío Páez

Peter B. Smith

Elza María Techio

Jorge Yamamoto 


\section{REVISTA DE PSICOLOGÍA}

ISSN 0254-9247

Vol. XXV (2), 2007

\section{CONTENIDO}

INTRODUCCIÓN

\section{ARTÍCULOS}

Fathali M. Moghaddam, Naomi Lee y Rom Harré. Psychology is social: Exploring universals in performance capacity and performance style

Peter B. Smith. Towards studies of organizational behaviour with greater local relevance

Jorge Yamamoto y Ana Rosa Feijoo. Componentes émicos del bienestar. Hacia un modelo alternativo de desarrollo

María Ángeles Bilbao, Elza María Techio y Darío Páez. Felicidad, cultura y valores personales: estado de la cuestión y síntesis meta-analítica

Ana Maria Anguas-Wongy David Matsumoto. Reconocimiento de la expresión facial de la emoción en mexicanos universitarios 
Agustín Espinosa, Alicia Calderón-Prada, Gloria Burga y Jessica Güimac. Estereotipos, prejuicios y exclusión social en un país multiétnico: el caso peruano 
Revista de Psicología Vol. XXV (2), 2007 (ISSN 0254-9247)

\title{
Componentes émicos del bienestar. Hacia un modelo alternativo de desarrollo ${ }^{1}$
}

\author{
Jorge Yamamoto $^{2}$ y Ana Rosa Feijoo ${ }^{3}$ \\ Pontificia Universidad Católica del Perú
}

Se presenta una propuesta alternativa a los modelos establecidos de bienestar y desarrollo a través de una metodología émica y post hoc. Esta se aplica a un corredor migratorio desde comunidades rurales hasta urbano marginales. Se aplicó una batería psicométrica desarrollada para este estudio a 550 participantes y una versión modificada del análisis factorial confirmatorio para satisfacer las demandas de teorización post-hoc del estudio. Se proponen modelos alternativos de necesidades universales, satisfacción subjetiva de vida, recursos y valores. Se encuentra una estructura de tres factores de personalidad. Los resultados son discutidos en relación con las teorías establecidas desde el referente europeo y estadounidense y se reflexiona sobre sus perspectivas para el desarrollo.

Palabras clave: bienestar, desarrollo, necesidades, metas, recursos, valores, personalidad, psicología transcultural, etnopsicología, comunidades rurales, comunidades urbano marginales.

\section{Emic components of well-being. Towards an alternative development model}

An alternative model of well-being and development using an emic and post-hoc approach is presented. This method was applied to a non-educated corridor sample which includes rural and urban shantytowns $(\mathrm{n}=550)$. A psychometric wellbeing battery was developed for this study. Analytical technique includes a modified version of Confirmatory Factor Analysis in order to meet the demands of the post-hoc theorisation rationale. Alternative models of universal needs, subjective life satisfaction, resources, and values are presented. A three factor personality solution is described. Discussion emphasises the advantages of these results compared to theories and approaches from Europe and United States in theoretical and empirical terms. Implications for development are also discussed.

Keywords: Well-being, development, needs, goals, resources, values, personality, cross-cultural psychology, ethnopsychology, rural communities, urban communities. 

El bienestar y la felicidad han motivado una permanente reflexión desde los albores de la reflexión filosófica. Durante los últimos años, la comunidad académica está prestando especial atención para su estudio científico (Diener, Oishi \& Lucas, 2003; Huppert, Baylis \& Keverne, 2005; Kahneman, Diener \& Schwarz, 1999; Ryan \& Deci, 2001). El modelo moderno de sociedad ha traído un impresionante desarrollo de la ciencia y la tecnología, entre otros. Sin embargo, el desarrollo económico, factor central del modelo, está restringido para un sector limitado de la población mundial; no se ha alcanzado la equidad ni se ha eliminado la pobreza. En los países ricos, paradójicamente, se encuentran bajos índices de felicidad (Marks, Abdallah, Simms \& Thompson, 2006), menor bienestar, menor salud mental, menor ajuste y más transtornos de comportamiento (Kasser \& Ryan, 1993, 1996). El sueño moderno no es un sueño para todos pero, cuando cumplido, se vuelve una pesadilla en la arena del bienestar.

La discusión sobre la influencia de la cultura, en particular de los valores del investigador, tanto en la teorización como en la investigación, es un tema aceptado. Existe considerable evidencia sobre la influencia

1 Este trabajo fue parte del programa del Economic and Social Research Council (ESRC), Reino Unido, "Grupo de investigación en bienestar en países en desarrollo". Los autores agradecen a los investigadores de campo Lidia Carhuallanqui, Edwin Páucar, Martín Jaurapoma, Miguel Obispo, Percy Reyna y Maribel Arroyo, también a Alejandro Lazarte, James Copestake, José Luis Álvarez y Teófilo Altamirano, y a la Universidad Nacional del Centro y la Dirección Académica de Investigación (DAI) de la PUCP.

2 Professor asociado del Departamento de Psicología de la PUCP e investigador del grupo Wellbeing in Developing Countries de la Universidad de Bath, Reino Unido. Dirección postal: Departamento de Psicología, Pontificia Universidad Católica del Perú, Av. Universitaria 1801, Lima 32, Perú. Correo electrónico: jyamamo@pucp.edu.pe

3 Investigadora del Centro de Investigación Sociológica, Antropológica y Económica de la PUCP y del grupo de investigación Wellbeing in Developing Countries. Dirección postal: Victor Criado Tejada 2857, Urb. Elio, Lima 1, Perú. Correo electrónico: feijoo.ar@pucp.edu.pe 
de la cultura en el bienestar (Diener, Oishi \& Lucas, 2003; Schimmack, Radhakrishnan, Oishi, Dzokoto \& Ahadi, 2002; Stenberg \& Grigorenko, 2005; Suh, Diener, Oishi \& Triandis, 1998). Esta influencia no se limita a la muestra de los estudios y sus respectivas poblaciones, se manifiesta también en los investigadores que diseñan e interpretan los estudios. La actividad académica puede caer en una interpretación subjetiva de los objetos y sujetos de estudio, más que una descripción fidedigna y un análisis riguroso, pudiendo caer en los límites de una interpretación antojadiza y caricaturesca (para una reflexión clásica, ver Said, 1978).

El presente estudio analiza el bienestar desde una perspectiva alterna a los métodos y teorías establecidas desde los auto-denominados países del primer mundo, planteando una alternativa que controle la imposición cultural hacia la compleja y distinta realidad de los países denominados del tercer mundo. Esto resulta particularmente importante. Habida cuenta de la evidencia del bajo bienestar en países desarrollados y del alto bienestar en países en vías de desarrollo en América Latina y el Caribe (Kasser \& Ryan, 1993, 2001; Marks, Abdallah, Simms \& Thompson, 2006), la imposición podría llevar a la perpetuación de teorías basadas en axiomas que están en la raíz del bajo bienestar global. No pretendemos proponer una reacción ideológica de oposición, tan solo controlar las ideologías, mentalidades y cultura de los investigadores, sea de donde provengan, en el afán de reducir la opacidad entre los patrones del comportamiento de la población y la elaboración académica. Una teoría que parte de la identificación de las categorías émicas, avanzando en la identificación de patrones, hacia la teorización post-hoc (Pedhazur \& Pedhazur, 1991) basada en la realidad. Si bien este problema no es nuevo, el reto está pendiente. Este estudio no solamente tuvo que controlar nuestros propios sesgos, con métodos y verificaciones cruzadas. Antes, en el marco de un proyecto internacional, tuvimos que defender de forma firme y permanente nuestra propuesta tercermundista frente a posiciones teóricas y metodológicas establecidas en Europa y Norteamérica. Adicionalmente, este problema no se mani- 
fiesta solamente en la actividad académica. Los programas de desarrollo suelen imponer los valores y concepciones de desarrollo propios de las agencias de desarrollo cuando, por un principio de respeto intercultural y de eficacia de intervención, se debiera de considerar los valores y concepciones propias de las comunidades meta de las intervenciones (Yamamoto, 2005, 2006). La metodología que planteamos podría aportar sobre un importante problema académico y de intervención social comunitaria.

\section{Componentes del bienestar}

En relación con los componentes del bienestar, existe cierto consenso en que el bienestar implica necesidades (Brunstein, 1993; Emmons, 2003; Locke, 2002; Schmuck \& Sheldon, 2001; Sheldon, Kasser, Smith $\&$ Share, 2002) entendidas como un conjunto de requerimientos de tendencia universal que deben ser satisfechos. La economía enfatiza el papel de los recursos como medios para alcanzar el bienestar. La satisfacción con la vida es considerada como el componente cognitivo del bienestar hedónico (Ryan \& Deci, 2001) y es definida como el grado en el cual la persona se encuentra subjetivamente complacida con su vida vista desde una perspectiva general (Diener, 1984; Diener, Emmons, Larsen \& Griffin, 1985). Diversos estudios coinciden en la importancia de la personalidad en el bienestar, siendo esta su mejor predictor (Diener, Oishi \& Lucas, 2003; Weiss, King \& Enns, 2002; Weiss, King, $\&$ Perkins, 2006). Asimismo, la evidencia empírica sugiere el importante papel de la cultura en general y los valores en particular (Diener, Oishi \& Lucas, 2003; Schimmack, Radhakrishnan, Oishi, Dzokoto \& Ahadi, 2002). En suma, entre los componentes fundamentales del bienestar, encontramos las necesidades, los recursos, la satisfacción con la vida, los valores y la personalidad.

Las necesidades son desarrolladas desde perspectivas teóricas que asumen dimensiones universales (por ejemplo, Doyal \& Gough, 1991; 
Maslow, 1943) las cuales cuentan con mayor o menor soporte empírico. Medir directamente las necesidades puede ser tendencioso y sesgado, sin embargo, podemos evaluar las metas concretas que las personas se proponen y a través de estadística multivariada, identificar las variables latentes que explican la varianza de esas metas encontradas. Estas variables latentes las denominaremos necesidades.

Los psicólogos olvidamos el papel de los recursos, sin embargo, los economistas tienden a generalizar su importancia, en algunos casos colocándolos como medios únicos necesarios para alcanzar la felicidad. En el presente estudio, definiremos los recursos como los medios para alcanzar las metas propuestas. El análisis de los mismos indicará el peso de lo económico versus lo no económico.

Entendemos la satisfacción de vida como la percepción de logro de las metas que la persona se propone. Esta definición tiene la ventaja de indicar el contenido de la satisfacción / insatisfacción, elemento fundamental para el diagnóstico y la intervención, en comparación con el modelo de satisfacción con la vida (Diener, Emmons, Larsen \& Griffin, 1985; Lewis, Shevlin, Bunting \& Joseph, 1995; Pavot \& Diener, 1993; Pavot, Diener, Colvin \& Sandvik, 1991).

La cultura y los valores suelen ser abordados desde dimensiones culturales específicas como el individualismo y el colectivismo (Diener, Oishi \& Lucas, 2003; Schimmack, Radhakrishnan, Oishi, Dzokoto \& Ahadi, 2002). El presente estudio abordará los valores desde una perspectiva más amplia que pueda rechazar, confirmar o precisar la validez de las diversas teorías de valores para la muestra del estudio. Definimos los valores como guías de comportamiento compartidas por un grupo que las considera universales y que funcionan como atajos evolutivos para la satisfacción de necesidades en determinados entornos. Es importante separar los valores de las formas social y políticamente deseables. Más aún, los valores pueden estar en contradicción con aquellas y los instrumentos de medición deben ser sensibles a discriminar esta interferencia. 
Habida cuenta de la validez intercultural del Modelo de Cinco Factores Robustos de Personalidad (MCFR) (McCrae \& Costa, 1997; McCrae et al., 2000) y especialmente de las limitaciones de recursos para conducir un estudio émico y post-hoc de personalidad, utilizaremos esta teoría, analizando de forma previa su validez para esta muestra.

\section{Metodología}

El sesgo del investigador, proceso que queremos controlar, se puede manifestar en la elección de los instrumentos, en la redacción de las preguntas de los instrumentos, en la teoría que la sustenta, en la interpretación de los resultados, entre otros. Para controlar el efecto de la teoría, asumimos una aproximación post-hoc (Hair, Anderson, Tatham $\&$ Black, 2004). No partimos de una teoría establecida, más bien, desarrollamos una metodología que parte de una descripción general de la realidad para ir identificando patrones y, así, llegar al plano teórico, contrastando los resultados para ver si estos brindan soporte a alguna teoría o se requiere plantear una nueva teoría. La literatura europea denomina a este enfoque grounded theory.

La primera fase del estudio se basó en la investigación etnográfica. En cada sitio de estudio, un investigador migró y se asentó durante el período del estudio que se extendió aproximadamente por tres meses. El primer mes se dedicó a desarrollar la etnografía del sitio, en forma de una anamnésis comunitaria, y entender la comunidad en su contexto e historia. Asimismo, este mes permitió ganar la confianza de la población, a fin de obtener respuestas más íntimas y profundas. La segunda fase del estudio consistió en una entrevista a profundidad de respuesta abierta que indagó sobre los siguientes componentes de bienestar: metas de vida, recursos para alcanzar estas metas, valores, momentos felices e infelices (Entrevista a Profundidad de Componentes Émicos de Bienestar / ECB) (Yamamoto, 2004). La tercera parte del estudio consistió en el desarrollo de una batería psicométrica de bienestar, cuya 
estructura e ítems fueron desarrollados sobre la base de los resultados del análisis de contenido de la segunda fase del estudio (Wellbeing in Developing Countries Quality of Life Dimension - Versión Perú / WQP) (Yamamoto \& Feijoo, 2005). En el presente artículo se presenta de forma general los resultados de la segunda fase del estudio. En documentos posteriores se analiza y presenta con profundidad cada uno de los aspectos de este estudio.

\section{Participantes}

Se realizó un muestreo basado en el sistema de corredor. Se identificó una vía de migración, desde pequeñas comunidades tradicionales, pasando por pueblos peri urbanos, hasta asentamientos urbano marginales. El centro del corredor se ubicó en el valle del Mantaro, iniciándose en comunidades rurales de Huancavelica, avanzando por centros peri urbanos y urbano marginales de Junín. Se incluyó una muestra en Huaycán, Lima, que recibe una fuerte migración de la zona. Adicionalmente, se consideró una comunidad de ceja de selva en Junín, que también recibe la migración de los habitantes del valle del Mantaro. El muestreo fue por cuotas. En cada sitio, la muestra fue proporcional a los barrios que la componen. En el caso de Huaycán, dado el tamaño de su población, se agruparon zonas por su semejanza y la muestra fue proporcional a estas zonas. La muestra se distribuyó de la siguiente forma: treinta participantes para la comunidad rural de ceja de selva, sesenta participantes para cada una de las dos comunidades rurales, cien participantes para cada uno de los dos sitios peri urbanos y cien participantes para cada uno de los dos sitios urbano marginales. La muestra total fue de 550 participantes. Un $47 \%$ de la muestra contaba solamente con educación primaria. El 27\% de la muestra no había completado la primaria. Así, esta muestra tiene la ventaja de representar mejor a la población, en comparación con los estudios basados en estudiantes universitarios. 


\section{Intrumentos}

Se desarrolló la Entrevista a Profundidad de Componentes Émicos de Bienestar (ECB) (Yamamoto, 2004). Es un protocolo de entrevista modular estructurado de respuesta abierta que indaga cinco áreas fundamentales: metas de vida, recursos, valores, episodios más felices y episodios más infelices.

\section{Técnica analítica}

Con el fin de identificar la estructura de los componentes del bienestar, se utilizó un procedimiento ad-hoc basado en el análisis factorial confirmatorio. Este procedimiento en su versión estándar debe estar sustentando en la teoría, de lo contrario, por cuestiones de azar, los resultados pueden arrojar coeficientes confirmatorios falsos, especialmente si se utilizan los índices de modificación (Hair, Anderson, Tatham \& Black, 2004). Esta condición contradice la naturaleza émica y post-hoc de este estudio. Con el fin de resolver esta paradoja, se realizaron análisis utilizando los índices de modificación hasta obtener una solución confirmatoria. Se obtuvieron varias soluciones siguiendo este procedimiento. Luego, se realizó este mismo procedimiento con una muestra equivalente para identificar el modelo robusto que subyace a las diferentes soluciones y muestras. De esta forma se redujo la probabilidad de un resultado confirmatorio falso. De forma adicional y de mayor importancia, los resultados fueron contrastados con los datos etnográficos y los resultados de las entrevistas a profundidad de la primera fase del estudio. Así, sustituimos el contraste con la teoría por el contraste con la realidad. Los análisis fueron conducidos utilizando los programas SPSS 14 y AMOS 5. 


\section{Resultados}

\section{Metas y necesidades}

Las metas fueron las variables observadas en el estudio. Definimos necesidades como las variables latentes de las metas. Se encuentran tres necesidades $\chi^{2}(32, N=500)=40.765, p=.138, C F I=.990, R M S E A=$ .023. 1) Lugar para vivir mejor, que tiene tres indicadores: vecindario limpio y bonito $(\lambda=.79)$, tranquilidad (sin delincuencia ni violencia; $\lambda=.64) \mathrm{y}$ salir adelante $(\lambda=.48)$. 2) Formar una familia, que describe dos indicadores: pareja o matrimonio $(\lambda=.79)$ e hijos $(\lambda=.77)$. 3) Desarrollo, ${ }^{1}$ que tiene como indicadores el trabajo asalariado $(\lambda=.55)$, la vivienda $(\lambda=.53)$, la educación para los hijos $(\lambda=.50)$, la salud y la alimentación $(\lambda=.50)$ y ser profesional $(\lambda=.38)$.

La necesidad de formar una familia es considerada por la teoría evolucionista como el eje de la vida (Darwin, 2004). Asimismo, psicólogos evolucionistas como Buss (2004) la consideran como motivación fundamental para el ser humano. Un lugar para vivir mejor se vincula directamente con un patrón de comportamiento también universal como la migración, habida cuenta que esta se da hacia un lugar en donde se percibe una vida mejor. El Perú describe fuertes patrones de migración interna y externa (Altamirano, 1988, 1992). En el resto del mundo la migración constituye también un fenómeno ubicuo. Aquellas pocas regiones donde no se encuentra una significativa migración, en el pasado la habrán tenido. Más aún, los estudios de las mutaciones del cromosoma Y proveen evidencia de la migración ancestral: el ser humano surgió en Etiopía y desde allí pobló todo el planeta (Wells, 2003). Estas dos necesidades son las más robustas. Cuando comparamos la muestra peruana con culturas y entornos diferentes como Bangladesh y Tailandia,

1 En su concepción moderna, no queremos decir que esta es nuestra concepción de desarrollo, sino la descrita por la muestra. Más aún, nuestras conclusiones apuntan a una crítica al modelo establecido de desarrollo. 


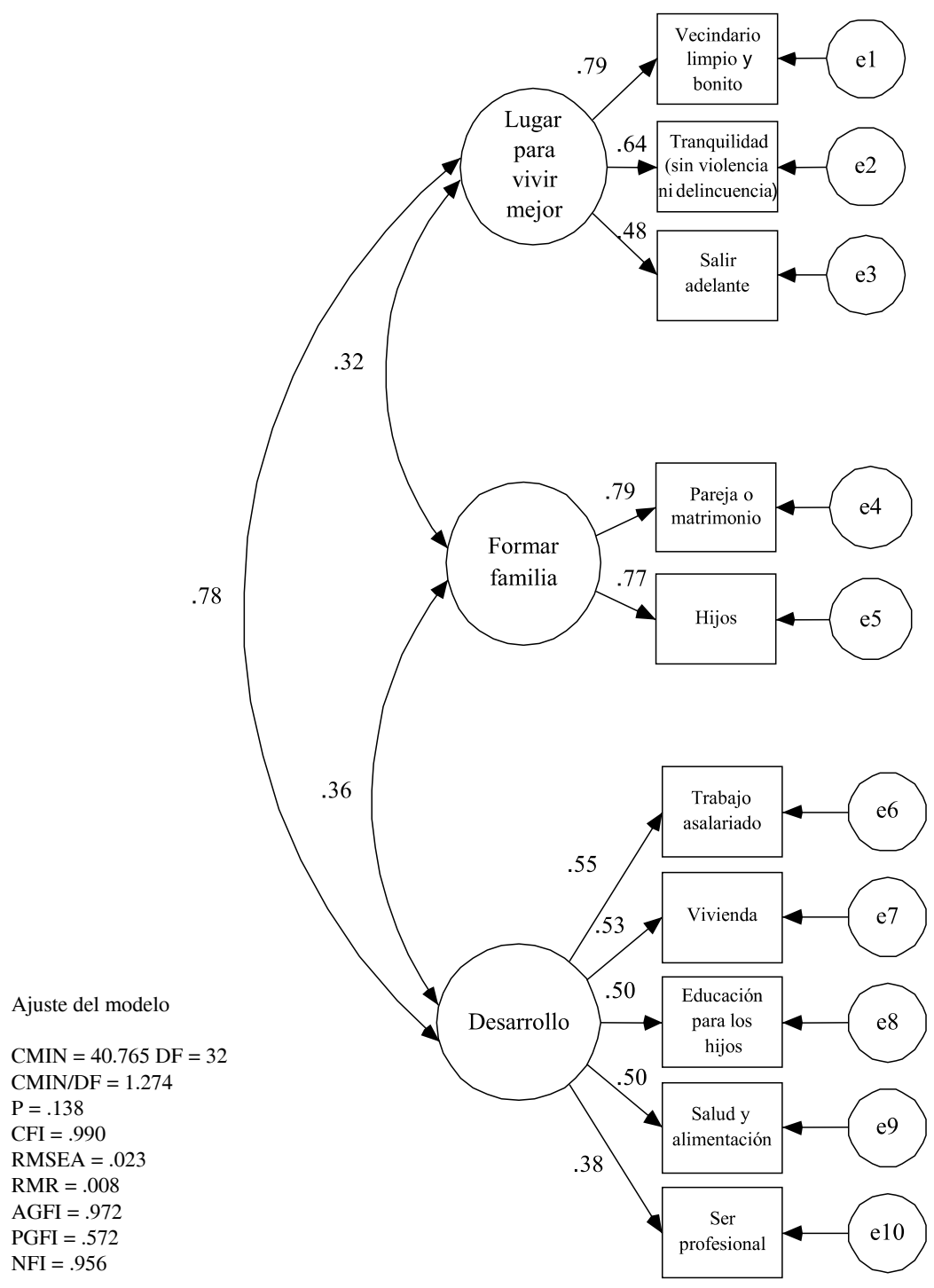

Figura 1. Análisis factorial confirmatorio de necesidades. 
la solución confirmatoria presenta solamente estos dos factores. ${ }^{2}$ Desarrollo, el tercer factor, describe la aspiración por la concepción moderna de desarrollo: trabajo, vivienda, educación, alimentación y salud. No se trata solamente de la supervivencia, describe connotaciones de mejora. La muestra cuenta con una educación limitada, sin embargo, alcanzar el nivel profesional aparece como aspiración. De igual forma, el trabajo asalariado es más una aspiración que una realidad. La necesidad de desarrollo es explicada por el sitio $F(6,543)=37.403, p<.001$ y es mayor en los sitios rurales. Esto se encuentra estrechamente vinculado a la migración, dado que esta se da no solamente a sitios en donde se percibe un mejor lugar para vivir, particularmente a sitios en donde se percibe un mayor desarrollo. La covarianza entre un lugar para vivir mejor y desarrollo es de .78 .

Así, estas necesidades describen la realidad del Perú, están soportadas por patrones de comportamiento en otras culturas y pueden explicar comportamientos que se remontan a la historia de la humanidad. Estudios con muestras en otras culturas permitirán avalar la universalidad de esta teoría de tres necesidades.

\section{Percepción de logro de metas y satisfacción de vida}

Las metas y sus necesidades latentes dirigen el comportamiento hacia su consecución, en el centro de los procesos motivacionales de la persona. Adicionalmente, el logro o frustración de esas metas define la satisfacción de vida. Dado que la percepción de logro de metas no es una estructura independiente en sí, sino supeditada a la estructura latente de metas, no hemos identificado la estructura latente de percepción de logro de metas, más bien, utilizando la estructura latente de metas, hemos evaluado la percepción de logro de cada meta. Así, la escala de percepción de logro de metas es una copia de la lista de indicadores de metas preguntando en cuanto a su percepción de logro.

2 Estos resultados serán presentados en un artículo próximo. 
La satisfacción de vida es el contraste entre las metas y la percepción de logro de esas metas. En el ámbito del análisis de segundo orden, definimos satisfacción con la vida como el contraste entre las necesidades y su percepción de logro. Este contraste puede describir una situación de insatisfacción (meta significativamente alta), de satisfacción (logro significativamente alto) o de equilibrio (sin diferencia significativa).

Los modelos establecidos de satisfacción con la vida definen el concepto como una evaluación general acerca de la vida, utilizando una escala de cinco ítems en esa dirección (Diener, Emmons, Larsen \& Griffin, 1985; Pavot \& Diener, 1993; Pavot, Diener, Colvin \& Sandvik, 1991). Consideramos que nuestro modelo tiene la ventaja de precisar las dimensiones del bienestar, a través de sus necesidades, la significación estadística de la satisfacción y el grado y dirección de esa satisfacción en cada una de sus áreas específicas. Esto subsume un diagnóstico que puede orientar de forma más precisa y productiva a las intervenciones individuales como comunitarias, así como servir de referente para la evaluación del impacto de las intervenciones. El modelo de satisfacción con la vida (Diener, Emmons, Larsen \& Griffin, 1985) se inserta en la tradición hedónica, que separa el bienestar en sus componentes cognitivos (satisfacción con la vida) y afectivos (balance hedónico) (Watson \& Tellegen, 1988) y define el bienestar como la búsqueda del placer y la evitación del displacer (Ryan \& Deci, 2001). La tradición eudaimónica propone que las metas son fundamentales para el bienestar y la concreción de estas puede motivar a la persona a pesar que estas impliquen displacer (Ryan \& Deci, 2001). Nuestra propuesta se inserta en un punto integrador. Considera las metas y está abierta a la evaluación del balance hedónico concomitante a la satisfacción, insatisfacción o equilibro. En un documento en elaboración, reportaremos los efectos emocionales (balance hedónico) de la satisfacción de vida. En el presente artículo presentamos los resultados de la satisfacción de vida de segundo orden, según sitio. Dada la heterogeneidad de la muestra, la generalización de un solo índice de satisfacción que no considere la diversidad de los sitios resulta carente de riqueza. 
Satisfacción con un lugar para vivir mejor

La muestra está insatisfecha con su lugar para vivir (diferencia $M$ $=-.367) F(1,549)=319.055, p<.001$. Los sitios rurales describen un patrón de satisfacción heterogéneo $F(6,543)=8.407, p<.001$. Dentro de estas diferencias, el análisis post hoc de Tukey señala que dos sitios ubicados en la misma área geográfica, con el mismo patrón cultural y con tamaños de población relativamente similares describen las diferencias más grandes. Los sitios peri urbanos y rurales describen patrones homogéneos. Los sitios peri urbanos describen equilibrio en la satisfacción y los sitios urbano marginales insatisfacción, con el margen más grande entre las expectativas y las metas. Ningún sitio describe satisfacción. Los resultados sugieren una mirada hacia lo moderno ${ }^{3}$, donde las poblaciones rurales estarían insatisfechas por la distancia con el ideal. Las poblaciones peri urbanas acceden en parte a esa modernidad, manteniendo sus adaptaciones rurales, creando una situación de equilibrio. Las poblaciones urbano marginales podrían perder esas adaptaciones, contrastar su situación con los referentes urbanos ricos y así generar un mayor grado de insatisfacción.

\section{Satisfacción con formar una familia}

Se encuentra un nivel general de satisfacción con la familia formada (diferencia $M=.312), F(1,549)=107.196, p<.001$. El sitio tiene efecto en la satisfacción con formar una familia $F(6,543)=8.407, p$ $<.001$. Sin embargo, el análisis post hoc de Tukey muestra un patrón que no puede ser explicado por la clasificación rural, peri urbano y urbano marginal. Estos datos están soportados por la fase cualitativa del estudio, donde la familia describe una alta estructuración y fuente de soporte y bienestar comparado con los estándares urbanos occidentales. La Figura 2 muestra el efecto de la edad en la satisfacción $F(5,535)=$ $10.677, p<.001$ en un proceso de satisfacción ideal; las metas siguen

3 La mirada hacia lo moderno de todas las comunidades está corroborada por los estudios cualitativos de la primera fase de esta investigación. 


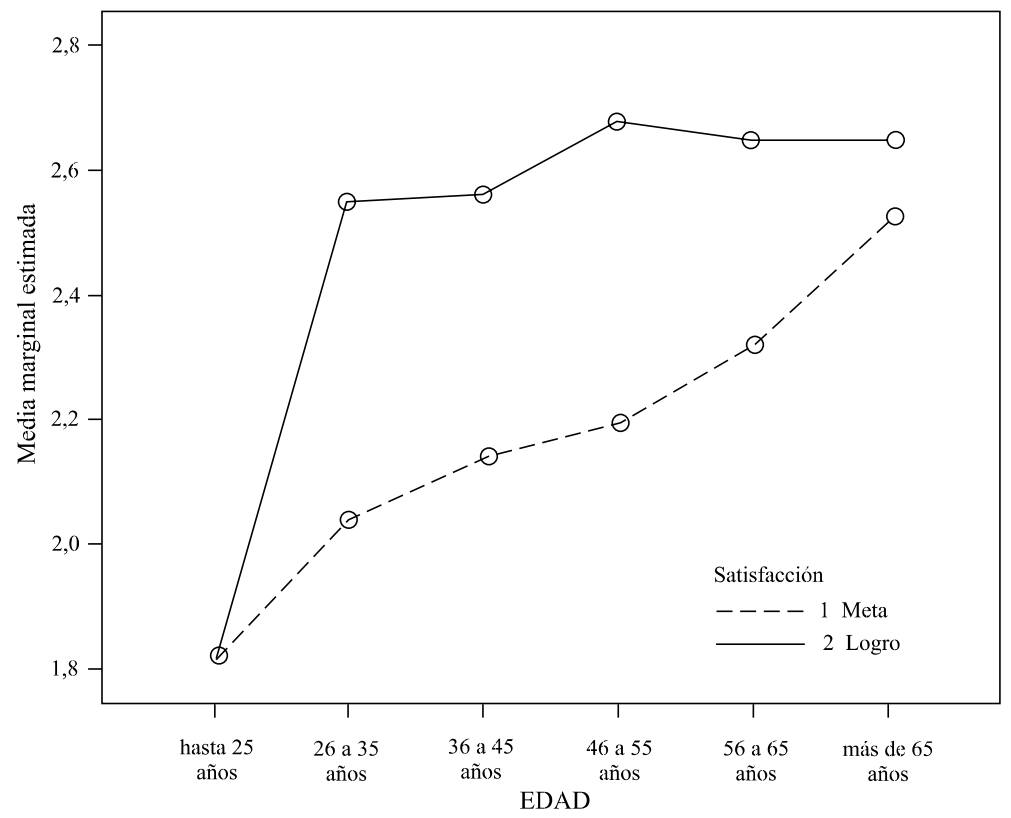

Figura 2. Anova series repetidas formar una familia por edad.

subiendo y la satisfacción se mantiene alta. Es decir, una satisfacción general y duradera, sin efecto de faja hedónica ni saturación.

Satisfacción con el desarrollo

La muestra está insatisfecha con el desarrollo moderno alcanzado (diferencia $M=-.696), F(1.549)=1688.70, p<.001$. El sitio tiene efecto en la insatisfacción $F(6,543)=p<.001$., Part. $\eta^{2}=.75$. Si bien no se encuentra un patrón claro entre sitios, la distancia de la insatisfacción sigue un patrón muy similar en los diferentes sitios, manteniendo una distancia relativamente constante entre la necesidad y la percepción de logro. Esto sugiere un proceso en el cual las metas modernas van elongándose manteniendo el umbral de insatisfacción. Este proceso ha 
sido previamente reportado como la faja hedónica sinfín (Brickman \& Campbell, 1971; Brickman, Coates \& Janoff-Bulman, 1978) y puede visualizarse en la Figura 3.

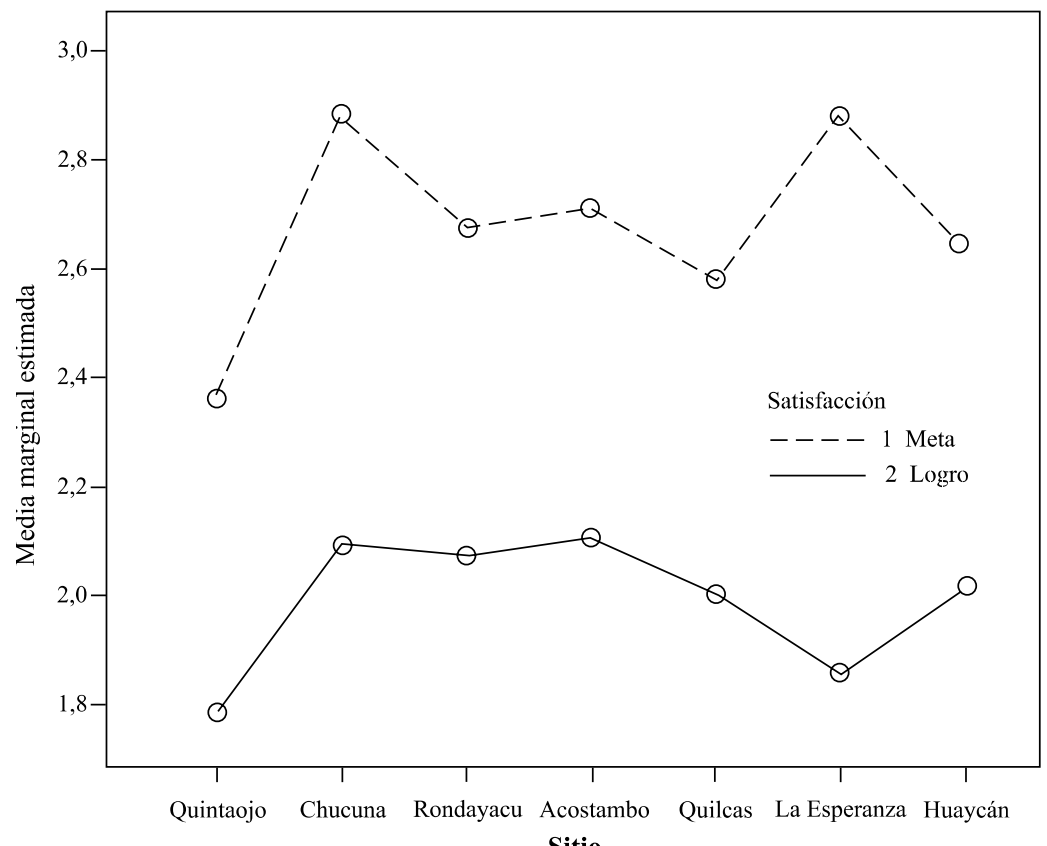

Sitio

Figura 3. Anova series repetidas desarrollo por sitio.

En palabras generales, la muestra está satisfecha con la necesidad de formar una familia. Está insatisfecha con su desarrollo, entendido como desarrollo moderno occidental. En cuanto al lugar para vivir mejor existe divergencia: os sitios urbano marginales están insatisfechos, los sitios peri urbanos están en equilibrio y existe diversidad en los sitios rurales. Si queremos hacer una generalización, podríamos señalar una insatisfacción en cuanto al lugar para vivir mejor y el desarrollo, en contraste con una satisfacción familiar. Estudios sobre el impacto de diferentes metas o necesidades en el bienestar señalan que las metas de 
carácter económico y extrínseco, como el desarrollo moderno y el lugar para vivir, tendrán un impacto neutro o negativo en el bienestar. En contraste, las metas intrínsecas, como la familia, tendrán un impacto positivo (Kasser \& Ryan, 1996, 2001; Sheldon, 2001, 2002; Sheldon \& Elliot, 1999; Sheldon \& Houser-Marko, 2001). Esto puede explicar los patrones de estudios mundiales de felicidad (Marks, Abdallah, Simms \& Thompson, 2006), en donde países como el Perú aparecen en puestos más altos de felicidad comparados con los países desarrollados que reciben los flujos migratorios del Perú y otras partes del tercer mundo. Aquí, por razones ajenas a la voluntad de las personas, no se pueden alcanzar las metas extrínsecas. Por razones culturales y probablemente vinculadas a la anterior, la familia constituye el centro de las satisfacciones. Habida cuenta que esta genera un mayor impacto en la felicidad, el resultante es un nivel de bienestar mayor. En contraste, en el mundo autodenominado desarrollado, se alcanzan las metas de desarrollo y de un buen lugar para vivir, en desmedro de formar una familia, ecuación que lleva a una resultante infelicidad.

\section{Recursos}

$\mathrm{El}$ análisis de recursos que presentamos no pretende ser exhaustivo en la medida que la economía ha desarrollado un sofisticado aparato teórico y metodológico para el estudio de los recursos materiales, cuya complejidad escapa del objeto de este estudio. Asimismo, el enfoque económico incorpora algunos supuestos que no asumimos. La economía clásica se basa en el supuesto que la toma de decisión es racional, tema que no es sostenible por las teorías psicológicas contemporáneas. Si bien es cierto que recientemente algunas aproximaciones desde la economía están cuestionando este axioma, la disciplina aún está embebida de este sesgo. Así, la economía estudia los recursos desde una perspectiva fundamentalmente material y, cuando se trata de introducir lo subjetivo, se realiza con ingenuidad teórica y metodológica. Definimos operacionalmente los recursos como los medios para obtener las metas que se plantean las personas. Así, en el protocolo de entrevista a 
profundidad ECB (Yamamoto, 2004), luego que el entrevistado lista sus metas de vida, se le pregunta, para cada una de ellas, qué medios utiliza para su realización. A través del análisis de contenido se depuró la lista, eliminando aquellos medios que a la vez eran metas, con el fin de evitar redundancia en el análisis estadístico. Se está preparando un análisis cualitativo sobre la relación redundante de las metas y los recursos. Sobre esta lista depurada se construyeron los ítems de la escala de recursos que forma parte de la batería psicométrica de bienestar WQP (Yamamoto \& Feijoo, 2005).

Los datos se analizaron de forma similar a las metas-necesidades, encontrando una solución unidimensional recursos $\left(\chi^{2}(14, N=531)\right.$ $=20.671, p=.110, C F I=.987, R M S E A=.030)$. Sus indicadores son: tener quién le preste (quién se refiere a una persona más que a una institución financiera) $(\lambda=.54)$, alquilar y arrendar $(\lambda=.57)$, tener quién le recomiende (para poder conseguir trabajo, por ejemplo) $(\lambda=.53)$, ser sujeto de herencia $(\lambda=.53)$, gestiones ante el gobierno (con el fin de obtener ayuda, por ejemplo, para la electrificación de la comunidad) $(\lambda=.49)$, ahorro (en dinero y en especies) $(\lambda=.56)$ y la migración $(\lambda=.42)$.

Este resultado enfatiza que los recursos materiales y no materiales están entrelazados. La lógica de mercado es insuficiente y hasta impertinente en este caso. Recuerda a los economistas que un análisis de los recursos como exclusivamente materiales es una reducción de la complejidad de la realidad social. Más aún, los modelos de desarrollo basados en el desarrollo económico carecen de sentido en estas realidades. También recuerda a los psicólogos que el análisis de los recursos como exclusivamente subjetivos es una reducción de la complejidad social. Más aún, un modelo de bienestar basado exclusivamente en variables subjetivas constituye un reduccionismo psicologista que debemos evitar. En este desbalance, probablemente, los economistas hayan tomado más conciencia de la importancia de lo subjetivo que los psicólogos de la importancia de los procesos vinculados a las variables materiales. 


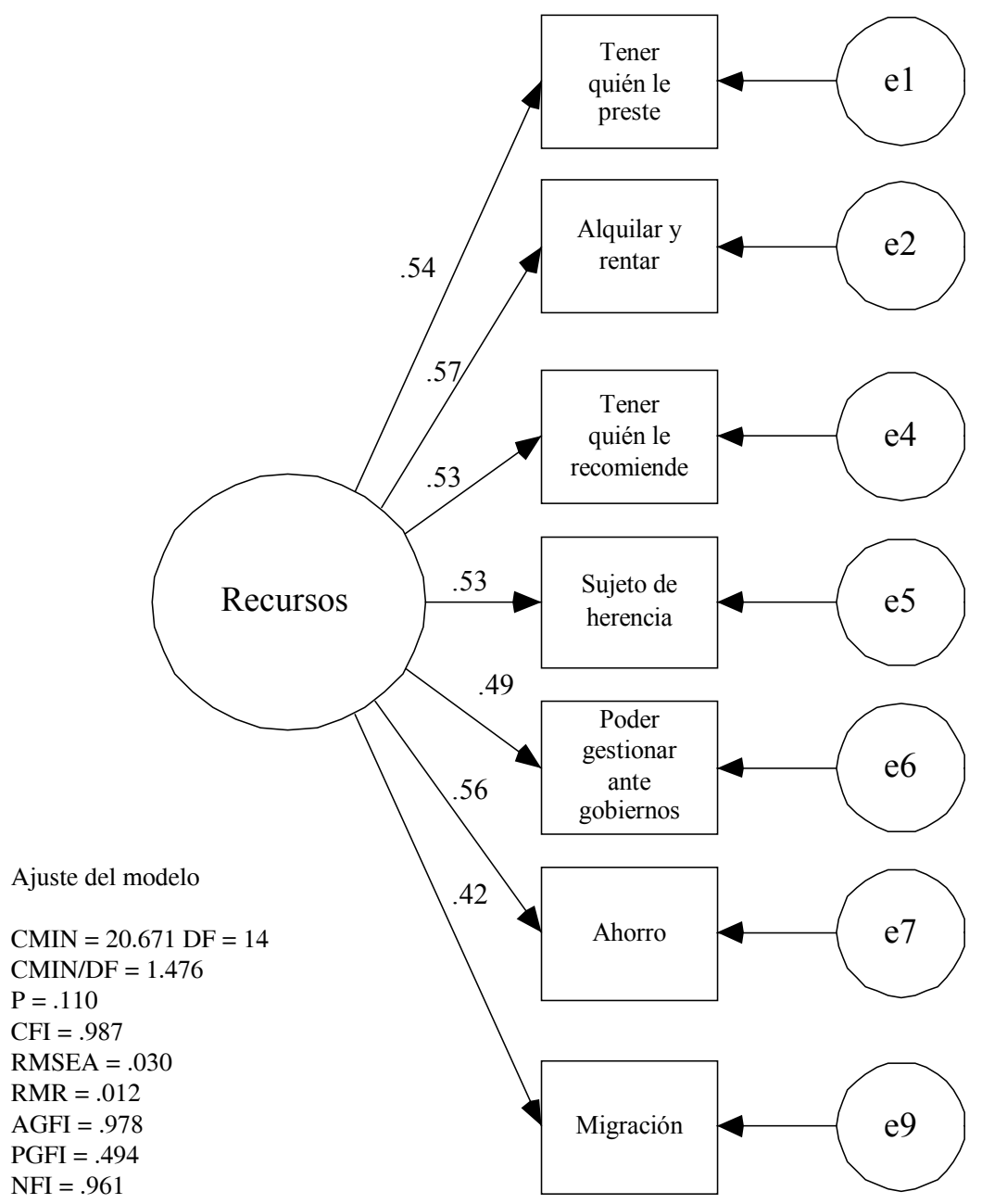

Figura 4. Análisis factorial confirmatorio de recursos. 


\section{Valores}

Se encuentra una solución de dos factores $\left(\chi^{2}(4, N=522)=1.237\right.$, $p=.872, C F I=1.0, R M S E A<.001)$ : colectivismo e individualismo. Diferentes autores han propuesto esta clasificación (Markus \& Kitayama, 1991; Suh, Diener, Oishi \& Triandis, 1998; Triandis, Bontempo, Villareal \& Asai, 1988) como orientaciones culturales en general tanto como valores en particular (Oishi, Diener, Suh \& Lucas, 1999). Nuestros resultados confirman estas orientaciones. Sin embargo, el contenido de los mismos varía significativamente.

El colectivismo está formado por tres indicadores: apoyo y consejo $(\chi=.89)$, compartir $(\lambda=.78)$ y organización vecinal y progreso individual $(\lambda=.44)$. Es interesante resaltar el carácter híbrido de los indicadores. El primero implica consejo, lo cual resalta el carácter no material del indicador, junto con apoyo, de connotación amplia. El tercero fusiona la organización vecinal con el progreso individual, sugiriendo que no se trata, como señalarían Markus y Kitayama (1991), que es una subordinación del individuo sobre el colectivo, sino una estrategia donde ambos niveles van de la mano. Esto se observa con claridad en las comunidades rurales. Dadas las condiciones geográficas y de población, donde no existe mano de obra ni subcontratación, la unión de la comunidad resulta importante para labores colectivas como la cosecha, la siembra, el mantenimiento de caminos o la construcción de casas. Así, el progreso individual no está contrapuesto sino fusionado a lo colectivo. Más novedosa es la configuración de los indicadores de individualismo: envidia $(\lambda=.99)$ y egoísmo $(\lambda=.84)$. El individualismo no es visto como el ideal de la autonomía sino, en la práctica, como un antivalor. Ambos factores están negativamente correlacionados. Es importante interpretar estos factores en su contexto. En el mundo urbano occidental, con relaciones anónimas y masivas y una fuerte competencia, una estrategia colectivista puede no tener la lógica que describimos para una comunidad rural. Este relativismo no resulta tan simple, habida cuenta de la evidencia que las relaciones 


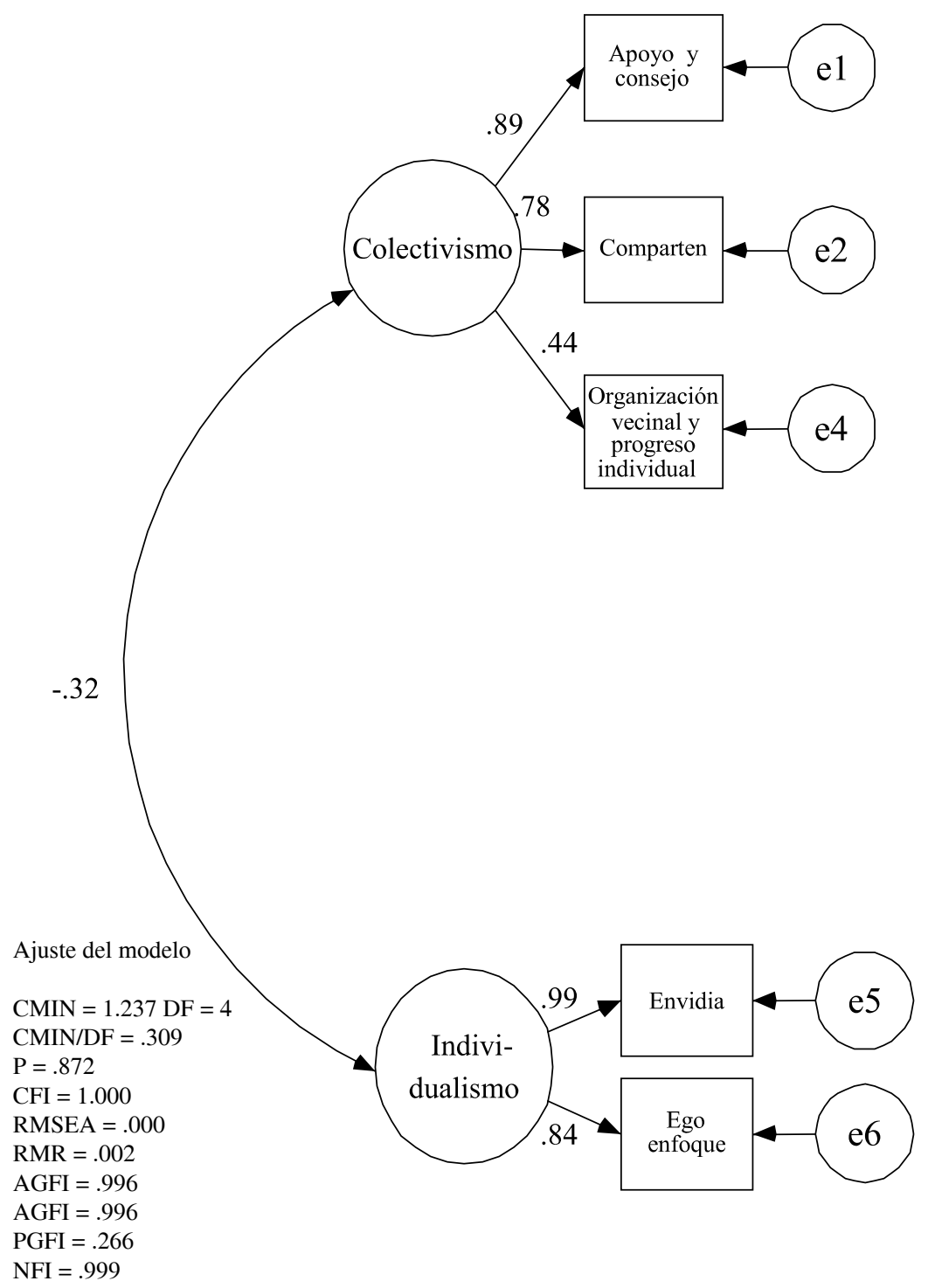

Figura 5. Análisis factorial confirmatorio de valores. 
interpersonales estrechas están vinculadas a un bienestar subjetivo superior (Buss, 2000; Kasser \& Ryan, 1996, 2001). Análisis con mayor profundidad sobre estos aspectos esclarecerán los correlatos subjetivos del individualismo y el colectivismo.

\section{Personalidad}

Abordar la personalidad desde la perspectiva émica y post-hoc que utilizáramos en este estudio excedía lejanamente los recursos disponibles. Sin embargo, la literatura era clara en mencionar la importancia de esta variable en el bienestar subjetivo (Diener, Oishi \& Lucas, 2003; Schimmack, Radhakrishnan, Oishi, Dzokoto \& Ahadi, 2002; Weiss, King \& Enns, 2002; Weiss, King \& Perkins, 2006). Así, utilizamos la prueba de personalidad de Kohlberg adaptada a muestras urbano marginales en el Perú por Calderón (Calderón, 2003). Se realizó un análisis factorial confirmatorio para probar el Modelo de Cinco Factores Robustos (MCFR) (McCrae \& Costa, 1997) de personalidad, sin encontrar evidencia que lo soportara $\left(\chi^{2}(735,322)=1393.526\right.$, $p<.001, C F I=.609, R M S E A=.053)$. Aplicamos el procedimiento de análisis confirmatorio modificado que utilizamos en los análisis previos encontrando tres factores $\left(\chi^{2}(41,322)=55.297, p=.67, C F I=961\right.$, $R M S E A=033)^{4}$. Estos resultados coinciden con estudios realizados en otras culturas en donde se encuentran soluciones diferentes a las planteadas por el MCFR (Di Blas, Forzi \& Peabody, 2000; Guanzon-Lapeña, Church, Carlota \& Katigbak, 1998; Saucier, Hampson, Goldberg \& Hampson, 2000).

El primer factor, mosca-ahuevado, describe cuatro indicadores: seguro $(\lambda=.40)$, perceptivo $(\lambda=.40)$, práctico $(\lambda=.57)$ y analítico $(\lambda=$ .61). El segundo factor, buena gente, cuenta también con cuatro indicadores: flexible $(\lambda=.46)$, ordenado $(\lambda=.46)$, desprendido $(\lambda=.58)$ y generoso $(\lambda=.48)$. Sociable y cálido es el tercer factor, sus indicadores

4 Nótese los índices de ajuste dentro del rango confirmatorio, pero menores en relación con las escalas émicas. 
son frío-cálido $(\lambda=.34)$, tímido-sociable $(\lambda=.63)$ y reservado-sociable $(\lambda=.63)$.

Los factores descritos no tienen una correspondencia operacional con el MCFR. Mosca-ahuevado incorpora indicadores de los factores de intelecto, estabilidad y conciencia. Buena gente cuenta con indicadores de extroversión y agradabilidad. Sociable y cálido describe componentes de agradabilidad y conciencia.

Un análisis etnográfico revela un posible sentido de estas diferencias. Por ejemplo, en un entorno donde la educación superior y la información global es restringida e innecesaria para el adecuado funcionamiento en ese entorno, el intelecto, según se describe en el quinto factor del MCFR, no tiene mucho sentido. Así, ser culto, erudito y complejo, por ejemplo, no resulta adaptativo. Más bien, el intelecto se definirá a través de otros indicadores pertinentes en el contexto de estas comunidades. Una inteligencia de carácter práctico, enfocada a resolver problemas más que a la abstracción. Las relaciones interpersonales son concretas, no existe el ciudadano y sus derechos, sino existen personas, con nombre, historia y familia; los problemas no son hipotéticos ni abstractos, son concretos, relativamente repetitivos en la historia dada la homogeneidad y rutina de la vida rural. Así, ser analítico, práctico, perceptivo y seguro ( $m o s c a$ ) serán indicadores que se referirán a ese intelecto como orientación analítica para la solución de problemas referidos a la satisfacción de necesidades, que en la práctica deja de ser aquello que en el mundo occidental llamamos intelecto. Más que ser agradable, lo cual connota un sentido de comportamiento apropiado para los segmentos urbanos ricos, se valora el ser buena gente, de connotación más popular y general, en donde la generosidad, factor fundamental para la vida interdependiente, juega un papel importante. Más que la extroversión, se valora el ser sociable y cálido; ser cool no es una forma adaptada en este contexto. 


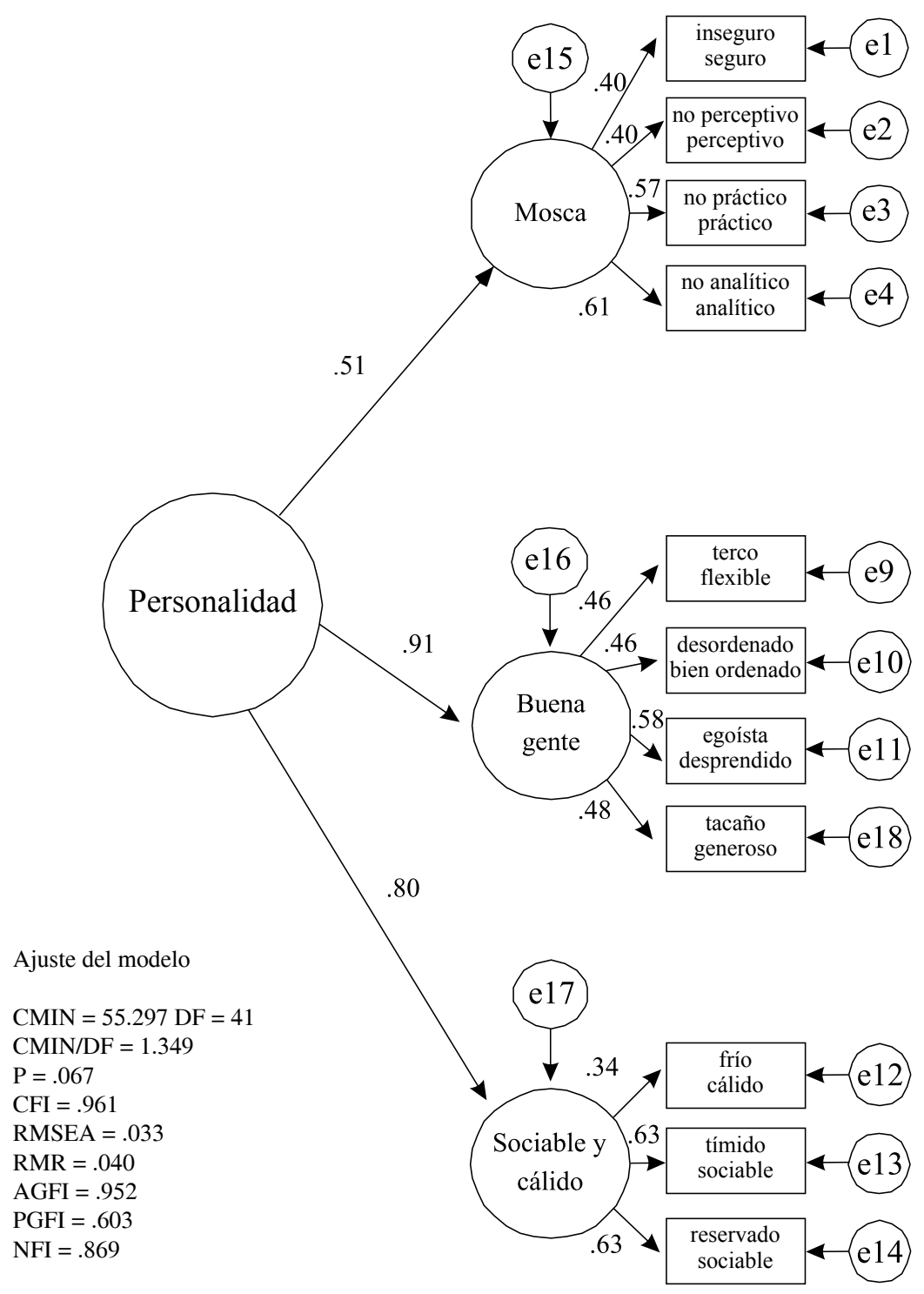

Figura 6. Análisis factorial confirmatorio de personalidad. 
A pesar de los resultados estadísticamente confirmatorios, se requieren estudios émicos adicionales dado el sesgo de los indicadores de partida que se han utilizado. De forma pre-confirmatoria, estos resultados sugieren que el MCFR señala en la dirección adecuada, es decir, que hay algo universal en cuanto al procesamiento cognitivo que media la satisfacción de necesidades, en cuanto a las formas apropiadas en cada contexto para la interacción social y en tanto la relación con uno mismo y con el submundo que le rodea. Sin embargo, la concreción de esos factores aún está sesgada por una tendencia occidental, habida cuenta de la carencia de un enfoque émico y de la utilización de muestras homogéneas (estudiantes universitarios de diferentes partes del mundo).

\section{Conclusiones}

Se encuentran tres necesidades: un lugar para vivir mejor, formar una familia y desarrollo. Estas necesidades están soportadas por la teoría evolucionista (Buss, 2004; Buss \& Kenrick, 1998; Darwin, 2004), sugiriendo sus profundas raíces en la constitución del ser humano. Los recursos para satisfacer estas necesidades son de carácter material y no material, estando entrelazados. El contraste entre estas necesidades y su percepción de logro nos brinda una forma válida y alternativa de conceptuar la satisfacción de vida. Se encuentra un nivel general de insatisfacción con el desarrollo. En cuanto a un lugar para vivir mejor, se encuentra insatisfacción en sitios urbano marginales, equilibrio en sitios peri urbanos y un patrón heterogéneo en sitios rurales. Se encuentra satisfacción con formar una familia con excepción de un sitio rural. Considerando evidencia previa de la relación de la satisfacción de metas intrínsecas versus extrínsecas (Kasser \& Ryan, 1993, 1996, 2001; Sheldon \& Elliot, 1999; Sheldon \& Houser-Marko, 2001), este patrón puede explicar la razón por la cual países latinoamericanos y caribeños describen índices de felicidad en contraste con los índices de infelicidad propios de los países autodenominados desarrollados, 
según las encuestas mundiales de felicidad (Marks, Abdallah, Simms \& Thompson, 2006).

Los recursos, definidos como los medios requeridos para alcanzar las metas de vida, describen elementos materiales, subjetivos y de redes sociales, los cuales están entrelazados y su disponibilidad en relación con la fijación de metas y su percepción de logro aparece como un mediador importante.

Encontramos dos factores de valores, individualismo y colectivismo. Estas dimensiones coinciden con estudios previos, sin embargo, los indicadores son diferentes. Colectivismo asume el apoyo objetivo y subjetivo, en una dinámica de compartir, uniendo el progreso individual con el colectivo. El individualismo es definido por dos indicadores: egoísmo y envidia.

Se encuentran tres factores de personalidad: mosca-ahuevado, buena gente y sociable y cálido. Estos factores no guardan relación con los indicadores de la teoría de los cinco factores robustos de personalidad.

\section{Discusión}

Los componentes analizados brindan una visión panorámica sobre el bienestar, incorporando elementos individuales, culturales, materiales y subjetivos. En una publicación en preparación, integraremos estos componentes en un modelo de bienestar a través de un modelo de ecuaciones estructurales. Asimismo, se requiere ampliar la muestra para avanzar hacia una teoría universal del bienestar. En próximas publicaciones reportaremos resultados en el Perú que incluyen muestras de la selva amazónica, de la costa rural, de la clase media urbana y de la clase alta urbana. Asimismo, estaremos presentando una muestra ampliada con comunidades rurales, peri urbanas y urbano marginales en Bangladesh y Tailandia, así como una muestra urbana en Colombia. 
La aproximación émica y post-hoc, junto con el trabajo en una muestra que no se limita a los estudiantes universitarios, ha permitido identificar modelos alternativos a las teorías de las necesidades establecidas, como la teoría de la auto-determinación (para una revisión, ver Ryan \& Deci, 2001) que propone la autonomía, la competencia y las relaciones como necesidades universales. Basados en la discusión sobre el soporte de la psicología evolucionista en relación con los tres factores que hemos encontrado (un lugar para vivir mejor, formar una familia y desarrollo), consideramos que los resultados presentados cubren aspectos más universales del comportamiento. Basados en la investigación etnográfica, consideramos que los resultados presentados reflejan con precisión la realidad de estos pueblos.

El contraste entre estas necesidades y su percepción de logro nos brinda una forma alternativa de conceptuar la satisfacción de vida. Brinda como ventaja la descripción de los componentes de la satisfacción, un nivel estadístico de satisfacción, equilibrio o insatisfacción y brinda un puente para la integración de la tradición hedónica y eudaimónica en la teoría del bienestar subjetivo. Esta diferencia puede repercutir tanto en el ámbito teórico como en el de la intervención, particularmente en el diagnóstico y evaluación de impacto.

Los economistas han simplificado la complejidad de la subjetividad de los recursos, mientras que los psicólogos hemos subvaluado la importancia de los recursos materiales. La medición de los recursos, tantos materiales como no materiales, brinda la oportunidad de evaluar su papel mediador en la satisfacción de vida y el bienestar.

El individualismo, visto desde una cultura no individualista, es definido como la envidia y el egoísmo. Así, el individualismo puede estar vinculado con el desarrollo económico (Hofstede \& Bond, 1984) pero, a la vez, al reducir la red de soporte e interacción social y estar esta vinculada al bienestar (Kasser \& Ryan, 1996, 2001; Sheldon \& Elliot, 1999; Sheldon \& Houser-Marko, 2001; Sheldon, Kasser, Smith 
\& Share, 2002), el individualismo puede contribuir a la reducción del bienestar y el colectivismo a su incremento.

Los tres factores de personalidad encontrados no guardan relación con los indicadores del MCFR (McCrae \& Costa, 1997; McCrae et al., 2000). Sin embargo, más allá de la discrepancia de los indicadores, los resultados sugieren que se tratan de dimensiones que van en la misma dirección. Por ejemplo, la dimensión de intelecto en el MCFR tiene una orientación a la cultura y la abstracción, forma de intelecto requerida para la vida en el mundo moderno. En contraste, hemos encontrado que mosca-ahuevado supone una inteligencia práctica y resiliente, característica importante para la vida en los entornos rurales, peri urbanos y urbano marginales. Así, en el ámbito de los indicadores, existe una diferencia significativa, pero en el plano de las dimensiones generales, se sugiere una similitud. Estudios posteriores deberían investigar las dimensiones de personalidad por debajo de las diferencias interculturales, especialmente entre muestras educadas y comunidades al margen de la socialización global.

Luego de analizar los componentes del bienestar podemos ensayar una definición preliminar, entendiéndola como el proceso de satisfacción de necesidades universales que se inicia con la fijación de metas, continúa con el acceso a los recursos para su concreción, el cual lleva a la percepción de logro de las mismas. La fijación de metas está vinculada a los valores y la personalidad. La percepción de su logro también estará vinculada a los valores y la personalidad.

Los afectos positivos y negativos serían concomitantes al estado del proceso. Por tanto, funcionarían desde una lógica motivacional, activándose en su carencia y desactivándose en su satisfacción. La potencia motivacional del bienestar estaría vinculada a los sentimientos extremos de felicidad e infelicidad. La segunda estaría vinculada a la evitación de estados que impidan el funcionamiento del individuo y sus grupos significativos, mientras que los primeros buscarían la concre- 
ción de metas positivas. Habida cuenta del enorme tiempo que implica la concreción de metas en el ser humano en comparación con otras especies, los estados de infelicidad y felicidad, como procesos de motivación, tendrían que haberse alongado proporcionalmente. Por tanto, el ser humano buscaría frenéticamente la felicidad, pero esta resultaría escurridiza por no ser un fin sino un afecto concomitante y efímero a la consecución de una meta y esta, por efecto motivacional, se debilitaría una vez alcanzada. Sobre esto último existe interesante evidencia desde la neuroimagen y otras aproximaciones (Marc, Paul \& Daniel, 2004; Myers \& Siegel, 1985; Nathan, Terry, Stephen \& Terry, 2005; Ranieri \& Zeiss, 1984; Walter \& Marc, 2006), en relación con la teoría de los procesos opuestos (Solomon, 1980). Asimismo, de forma más intensa, las personas buscamos evitar la infelicidad cuando esta, en su oscuridad, habría adquirido dimensiones sin paralelo en la evolución. Esta teoría requiere evidencia adicional que la sustente, la cual estaremos reportando próximamente.

El post-modernismo proclama el fracaso del modelo moderno de sociedad. El estudio científico de la felicidad lo operacionaliza, virando la atención del autodenominado mundo desarrollado (más precisamente mundo desarrollado económicamente y subdesarrollado en bienestar subjetivo), hacia el mundo denominado subdesarrollado (más precisamente el mundo de motivación económica secundaria y orientación al bienestar subjetivo). La investigación émica y post-hoc en sociedades marginales, voluntaria o involuntariamente, del proceso de desarrollo global, brinda una alternativa prometedora para la comprensión del bienestar y para el planteamiento de modelos alternativos de desarrollo o, quizá más precisamente, de antidesarrollo para un mundo al revés. 


\section{Referencias}

Altamirano, T. (1988). Cultura andina y pobreza urbana: Aymaras en Lima Metropolitana. Lima: Fondo Editorial de la PUCP.

Altamirano, T. (1992). Exxodo: peruanos en el exterior. Lima: Fondo Editorial de la PUCP.

Brickman, P. \& Campbell, D. T. (1971). Hedonic relativism and planning the good society. En M. H. Apley (Ed.), Adaptation-level theory: A symposium (pp. 287-302). Nueva York: Academic Press.

Brickman, P., Coates, D. \& Janoff-Bulman, R. (1978). Lottery winners and accident victims: Is happiness relative? Journal of Personality and Social Psychology, 36(8), 917-927.

Brunstein, J. C. (1993). Personal goals and subjective well-being: A longitudinal study. Journal of Personality and Social Psychology, 65(5), 1061-1070.

Buss, D. (2000). The evolution of happiness. American Psychologist, 55(1), 15-23.

Buss, D. (2004). Evolutionary psychology: The new science of the mind (2a. ed.). Boston: Pearson.

Buss, D. \& Kenrick, D. T. (1998). Evolutionary social psychology. En D. Gilbert, S. Fiske \& G. Lindzey (Eds.), The handbook of social psychology (Vol. 2, pp. 982-1026). Boston: Mc Graw Hill.

Calderón, A. (2003). Satisfacción marital desde la perspectiva de la psicologia evolutiva en parejas urbano-marginales de Lima. Tesis de licenciatura no publicada, Pontificia Universidad Católica del Perú, Lima.

Darwin, C. (2004). The origin of species. Nueva York: Barnes and Noble.

Di Blas, L., Forzi, M. \& Peabody, D. (2000). Evaluative and descriptive dimensions from Italian personality factors. European Journal of Personality, 14(4), 279-290.

Diener, E. (1984). Subjective well being. Psychological Bulletin, 95(3), 542-575. 
Diener, E., Emmons, R. A., Larsen, R. J. \& Griffin, S. (1985). The Satisfaction With Life Scale. Journal of Personality Assessment, 49(1), 71-75.

Diener, E., Oishi, S. \& Lucas, R. (2003). Personality, culture and subjective well-being: Emotional and cognitive evaluations of life. Annual Review of Psychology, 54, 404-425.

Doyal, L. \& Gough, I. (1991). A theory of human need. Houndmills, Reino Unido: MacMillan.

Emmons, R. A. (2003). Personal goals, life meaning, and virtue: Wellsprings of a positive life. En C. L. M. Keyes \& J. Haidt (Eds.), Flourishing: Positive psychology and the life well-lived (pp. 105128). Washington, DC: American Psychological Association.

Guanzon-Lapeña, M. A., Church, A. T., Carlota, A. J. \& Katigbak, M. S. (1998). Indigenous personality measures: Philippine examples. Journal of Cross-Cultural Psychology, 29(1), 249-270.

Hair, J. F., Anderson, R. E., Tatham, R. L. \& Black, W. C. (2004). Análisis multivariante (5a. ed.). Madrid: Prentice Hall Iberia.

Hofstede, G. \& Bond, M. H. (1984). Hofstede's culture dimensions: An independent validation using Rokeach's Value Survey. Journal of Cross-Cultural Psychology, 15(4), 417-433.

Huppert, F. A., Baylis, N. \& Keverne, B. (Eds.). (2005). The science of well-being. Nueva York: Oxford University Press.

Kahneman, D., Diener, E. \& Schwarz, N. (Eds.). (1999). Well-being: The foundations of hedonic psychology. Nueva York: Russell Sage Foundation.

Kasser, T. \& Ryan, R. M. (1993). A dark side of the American dream: Correlates of financial success as a central life aspiration. Journal of Personality and Social Psychology, 65(2), 410-422.

Kasser, T. \& Ryan, R. M. (1996). Further examining the American dream: Differential correlates of intrinsic and extrinsic goals. Personality and Social Psychology Bulletin, 22(3), 280-287.

Kasser, T. \& Ryan, R. M. (2001). Be careful what you wish for: Optimal functioning and the relative attainment of intrinsic and extrinsic goals. En P. Schmuck \& K. M. Sheldon (Eds.), Life goals and 
well-being: Towards a positive psychology of human striving (pp. 116-131). Ashland, OH: Hogrefe \& Huber.

Lewis, C. A., Shevlin, M. E., Bunting, B. P. \& Joseph, S. (1995). Confirmatory factor analysis of the Satisfaction With Life Scale: Replication and methodological refinement. Perceptual and Motor Skills, 80(1), 304-306.

Locke, E. A. (2002). Setting goals for life and happiness. En C. R. Snyder \& S. J. López (Eds.), Handbook of positive psychology (pp. 299-312). Nueva York: Oxford University Press.

Marc, R. L., Paul, K. \& Daniel, M. L. (2004). Affect responses to acute bouts of aerobic exercise: A test of opponent-process theory. Journal of Sport Behavior, 27(4), 330.

Marks, N., Abdallah, S., Simms, A. \& Thompson, S. (2006). The unhappy planet index. Londres: New Economics Foundation.

Markus, H. R. \& Kitayama, S. (1991). Culture and self: Implications for cognition, emotion, and motivation. Psychological Review, 98, 224-253.

Maslow, A. (1943). A theory of human motivation. Psychological Review, 50, 370-396.

McCrae, R. R. \& Costa, P. T. (1997). Personality trait structure as a human universal. American Psychologist, 52(5), 509-516.

McCrae, R. R., Costa, P. T., Ostendorf, F., Angleitner, A., Hrebickova, M., Avia, M. D. et al. (2000). Nature over nurture: Temperament, personality, and life span development. Journal of Personality and Social Psychology, 78(1), 173-186.

Myers, H. H. \& Siegel, P. S. (1985). The motivation to breastfeed: A fit to the opponent-process theory. Journal of Personality and Social Psychology, 49(1), 188-193.

Nathan, A. B., Terry, A. B., Stephen, H. W. \& Terry, M. L. (2005). Adaptation-level theory, opponent process theory, and dispositions: An integrated approach to the stability of job satisfaction. Journal of Applied Psychology, 90(6), 1.

Oishi, S., Diener, E., Suh, E. \& Lucas, R. E. (1999). Value as a moderator in subjective well-being. Journal of Personality, 67(1), 157-184. 
Pavot, W. \& Diener, E. (1993). Review of the Satisfaction With Life Scale. Psychological Assessment, 5(2), 164-172.

Pavot, W. G., Diener, E., Colvin, C. R. \& Sandvik, E. (1991). Further validation of the Satisfaction With Life Scale: Evidence for the cross-method convergence of well-being measures. Journal of Personality Assessment, 57(1), 149-161.

Pedhazur, E. J. \& Pedhazur, L. (1991). Measurement, design and analyses. An integrated approach. Nueva Jersey: Lawrence Erlbaum.

Ranieri, D. J. \& Zeiss, A. M. (1984). Induction of depressed mood: A test of opponent-process theory. Journal of Personality and Social Psychology, 47(6), 1413-1422.

Ryan, R. \& Deci, E. (2001). On happiness and human potentials: A review of research on hedonic and eudaimonic well-being. Annual Review of Psychology, 52, 141-166.

Said, E. W. (1978). Orientalism. Nueva York: Vintage Books.

Saucier, G., Hampson, S. E. \& Goldberg, L. R. (2000). Cross-language studies of lexical personality factors. En S. E. Hampson (Ed.), Advances in personality psychology (Vol. 1, pp. 1-36). Filadelfia: Psychology Press.

Schimmack, U., Radhakrishnan, P., Oishi, S., Dzokoto, V. \& Ahadi, S. (2002). Culture, personality, and subjective well-being: Integrating process models of life satisfaction. Journal of Personality and Social Psychology, 82(4), 582-593.

Schmuck, P. \& Sheldon, K. M. (2001). Life goals and well-being: Towards a positive psychology of human striving. Ashland, $\mathrm{OH}$ : Hogrefe \& Huber.

Sheldon, K. M. (2001). The self-concordance model of healthy goal striving: When personal goals correctly represent the person. En P. Schmuck \& K. M. Sheldon (Eds.), Life goals and well-being: Towards a positive psychology of human striving (pp. 18-36). Ashland, OH: Hogrefe \& Huber.

Sheldon, K. M. (2002). The self-concordance model of healthy goal striving: When personal goals correctly represent the person. En E. L. Deci \& R. M. Ryan (Eds.), Handbook of self-determina- 
tion research (pp. 65-86). Rochester, NY: University of Rochester Press.

Sheldon, K. M. \& Elliot, A. J. (1999). Goal striving, need satisfaction, and longitudinal well-being: The self-concordance model. Journal of Personality and Social Psychology, 76(3), 482-497.

Sheldon, K. M. \& Houser-Marko, L. (2001). Self-concordance, goal attainment, and the pursuit of happiness: Can there be an upward spiral? Journal of Personality and Social Psychology, 80(1), 152-165.

Sheldon, K. M., Kasser, T., Smith, K. \& Share, T. (2002). Personal goals and psychological growth: Testing an intervention to enhance goal attainment and personality integration. Journal of Personality, 70(1), 5-31.

Solomon, R. L. (1980). The opponent-process theory of acquired motivation. The costs of pleasure and the benefits of pain. American Psychologist, 35(8), 691-712.

Sternberg, R. J. \& Grigorenko, E. L. (2005). Intelligence and culture: How culture shapes what intelligence means, and the implications for a science of well-being. En F. A. Huppert, N. Baylis \& B. Keverne (Eds.), The science of well-being (pp. 379-404). Nueva York: Oxford University Press.

Suh, E., Diener, E., Oishi, S. \& Triandis, H. C. (1998). The shifting basis of life satisfaction judgments across cultures: Emotions versus norms. Journal of Personality and Social Psychology, 74(2), 482-493.

Triandis, H. C., Bontempo, R., Villareal, M. J. \& Asai, M. (1988). Individualism and collectivism: Cross-cultural perspectives on self in-group relationships. Journal of Personality and Social Psychology, 54(2), 323-338.

Walter, R. B. \& Marc, R. L. (2006). Affect responses to acute bouts of aerobic exercise in fit and unfit participants: An examination of opponent-process theory. Journal of Sport Behavior, 29(2), 111. 
Watson, D. C. \& Tellegen, L. (1988). Development and validations of brief measures of positive and negative affect: The PANAS scales. Journal of Personality and Social Psychology, 54(6), 1063.

Weiss, A., King, J. E. \& Enns, R. M. (2002). Subjective well-being is heritable and genetically correlated with dominance in chimpanzees (Pan troglodytes). Journal of Personality and Social Psychology, 83(5), 1141-1149.

Weiss, A., King, J. E. \& Perkins, L. (2006). Personality and subjective well-being in orangutans (Pongo pygmaeus and Pongo abelii). Journal of Personality and Social Psychology, 90(3), 501-511.

Wells, S. (2003). The journey of man: A genetic odyssey. Nueva Jersey: Random House.

Yamamoto, J. (2004). El protocolo de entrevista a profundidad de componentes de bienestar. Manuscrito no publicado.

Yamamoto, J. (2005). Hacia una metodología de intervención con criterio intercultural: SNV amazonía. Agencia holandesa de cooperación internacional. Manuscrito no publicado.

Yamamoto, J. (2006). Análisis intercultural de conflictos: concepciones nativas de desarrollo y bienestar. Proyecto focal bosques. Consorcio IIAP - SNV. Manuscrito no publicado.

Yamamoto, J. \& Feijoo, A. R. (2005). La Escala Psicométrica de Bienestar WQP. Manuscrito no publicado.

Recibido 20 de abril, 2007 Aceptado 11 de octubre, 2007 